\title{
Lost, fiction kaléidoscopique
}

\section{Claire Cornillon}

\section{(2) OpenEdition}

Journals

Édition électronique

URL : http://journals.openedition.org/tvseries/1632

DOI : 10.4000/tvseries. 1632

ISSN : 2266-0909

Éditeur

GRIC - Groupe de recherche Identités et Cultures

\section{Référence électronique}

Claire Cornillon, «Lost, fiction kaléidoscopique », TV/Series [En ligne], Hors séries 1 | 2016, mis en ligne le 01 octobre 2016, consulté le 01 mai 2019. URL : http://journals.openedition.org/tvseries/1632 ; DOI : 10.4000/tvseries.1632

Ce document a été généré automatiquement le 1 mai 2019.

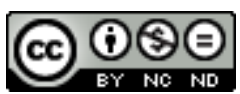

TV/Series est mis à disposition selon les termes de la licence Creative Commons Attribution - Pas d'Utilisation Commerciale - Pas de Modification 4.0 International. 


\title{
Lost, fiction kaléidoscopique
}

\author{
Claire Cornillon
}

1 Lost (ABC, 2004-2010) n'a cessé de questionner sa propre nature pour emmener les spectateurs là où ils ne pensaient pas pouvoir aller. Bien qu'il semble possible, à première vue, de dégager un glissement générique dans l'histoire de la série, du fantastique à la fantasy ${ }^{1}$, en passant par la science-fiction, il s'avère, à la lumière de la fin, que son univers fictionnel avait toujours été un monde de fantasy. C'est notre perception de la série qui a évolué au fur et à mesure de ses saisons. Lost est une fiction kaléidoscopique parce qu'elle est une fiction de l'éclatement apparent et de l'unité sous-jacente, voire souterraine. Or, la série est l'histoire de la construction d'une communauté, à travers cet itinéraire de transformation du point de vue. Elle n'est donc pas un jeu formel et énigmatique, mais bien un chemin initiatique, l'expérience d'un mystère auquel il faudra céder in fine pour s'approprier la série.

\section{Un glissement générique apparent}

2 La définition du fantastique, de la science-fiction et de la fantasy est une question très complexe, maintes fois discutée. Il me semble que ces trois catégories relèvent de démarches plutôt que de genres et j'ai essayé, ailleurs, de proposer quelques critères de différenciation $^{2}$ que je reprendrai ici. L'objet de cet article n'est pas néanmoins d'apposer une étiquette quelle qu'elle soit. Il s'agit de repérer trois modalités de la fiction qui aident à penser le fonctionnement de la série, mais que l'on peut qualifier comme on le souhaite. Je conserverai ici cependant les catégories traditionnelles de fantastique, de sciencefiction et de fantasy, en indiquant ce que je retiendrai de leur définition, puisque des références directes à la tradition de ces genres sont évoquées dans la série.

En effet, Lost charge son récit d'un ensemble de marques qui détournent le regard d'éléments qui se révèlent fondamentaux par la suite mais qui apparaissent pourtant dès le début de la série. Le prestidigitateur n'agit pas autrement : il attire notre attention quelque part afin de détourner notre regard de l'endroit où se joue réellement le tour. La spécificité de Lost est de nous montrer que nous regardions au mauvais endroit et donc de 
déconstruire l'illusion. Pour ce faire, elle dissémine une série de signes, de codes, que l'on peut rattacher à différents genres de l'imaginaire - le fantastique, la science-fiction et la fantasy. Ces codes de références s'entremêlent et sont plus ou moins dominants au cours de la série. À chaque fois que la série semble s'ancrer dans l'un de ces genres, une anomalie apparait et cette interprétation devient aporétique. Une nouvelle perspective naît alors qui fait changer notre regard. La fiction ne change pas tant de genre qu'elle nous lance volontairement des fausses pistes avant de s'affirmer pour ce qu'elle est.

La première fausse piste est certainement celle du récit "réaliste» car bien que l'existence des survivants après un tel crash soit surprenante, rien au départ ne laisse imaginer ce qui va suivre. Ce n'est qu'à la vingtième minute du pilote qu'un premier tournant est pris par la série, celui du fantastique. En effet les personnages entendent des bruits étranges dans la jungle, difficiles à identifier, dont certains sont métalliques. Il s'agit des bruits associés à la fumée noire mais le spectateur ne le sait pas encore. Le haut des palmiers s'agite, comme si une créature de grande taille se déplaçait, suggérant l'hypothèse selon laquelle l'île pourrait être habitée par un monstre, une sorte de King Kong des temps modernes. À ce stade, la question est légitime. Pourtant, aucune réponse n'est proposée. C'est d'ailleurs grâce à cette incertitude, cette hésitation parfaitement todorovienne ${ }^{3}$, que l'angoisse va surgir. L'angoisse est ce qui relève d'une menace inconnue ou invisible, à l'inverse de la peur qui naît face à un danger immédiat. Le fantastique se construit sur le surgissement d'un élément surnaturel, étrange, dans notre univers; cet élément fait obstacle cognitivement, il pose problème dans l'univers fictionnel, soit sur le mode de l'hésitation, soit sur le mode de la sidération ${ }^{4}$.

5 À partir de cette vingtième minute du pilote, différents éléments qui peuvent relever du surnaturel apparaissent. Leur nature est inconnue et leur existence même est sujette à caution. C'est notamment le cas de l'ensemble des visions des personnages, lorsque Jack voit, par exemple, son père décédé à plusieurs reprises. Il n'est pas possible alors de déterminer s'il s'agit de visions réelles ou d'hallucinations liées au traumatisme des personnages, d'autant que ces visions sont toujours, dans la narration, associées à des événements spécifiques du passé et à l'itinéraire présent du personnage. Cette hésitation entre réalité et hallucination est un topos du fantastique. D'autre part, la série s'appuie sur des codes visuels et musicaux qui relèvent du cinéma de genre, du cinéma fantastique voire du cinéma d'horreur, et invite ainsi les spectateurs à percevoir la fiction dans ce cadre d'interprétation. Ainsi, le monstre de fumée noire reste au départ invisible à l'écran parce qu'il apparait en caméra subjective ou en hors champ. Les personnages le voient ; les spectateurs le perçoivent seulement à travers le visage terrifié des personnages. Il s'agit là d'un procédé classique du cinéma ou des séries fantastiques, comme The X-Files (Fox, 1993-2002). Tout au long des premières saisons de Lost, certaines séquences fonctionnent comme des courts-métrages de cinéma de genre : ainsi la cabane de Jacob se fonde sur le modèle du film de maison hantée. De même, la mort du pilote de l'avion est tournée comme un film de monstre, dans une scène qui rappelle volontiers Jurassic Park (réal. Steven Spielberg, 1993) par le jeu sur le rythme, l'attente et la violence soudaine, les cris, les visages terrifiés, la menace invisible, la caméra qui bouge frénétiquement pour suggérer la panique, le filtre de couleur bleue et finalement le topos du jet de sang sur la vitre. L'image est saturée par ces codes: lors du premier visionnage de la série, il est impossible de voir autre chose, comme si Lost mettait en place un piège initiatique pour les spectateurs. 
6 Dans les trois premières saisons, ces derniers demeurent dans l'hésitation fantastique mais, par la suite, les éléments inexplicables deviennent de plus en plus nombreux et surtout commencent à se constituer en système. Il semble impossible de le décoder mais, de toute évidence, il existe. D’ailleurs, dans le pilote, le premier flashback apparaît juste après la première irruption de l'étrange - des bruits dans la forêt. Il instaure donc une rupture daens la narration et indique, par sa présence même, que des enjeux plus importants se cachent derrière le crash : ce dernier ne serait pas simplement un point de départ vers une intrigue qui relèverait du film d'horreur mais constituerait, en fait, un point d'arrivée ou d'étape. Dès lors qu'il y a système, le genre est soit celui de la sciencefiction, soit celui de la fantasy. Or c'est bien la science-fiction qui se présente comme une nouvelle fausse piste. Là encore des signes invitent progressivement à opérer ce changement de paradigme interprétatif, en particulier dans la saison 4. Certains sont anecdotiques, comme lorsque Locke donne à Ben le roman Valis de Philip K. Dick. Il ne s'agit pas d'un élément insignifiant puisque la science-fiction à laquelle il est fait référence appartient à une veine mystique et gnostique qui n'est pas sans résonance avec l'univers de la série, mais ce signe est micro-structurel ou ponctuel. Il y a par ailleurs d'autres signes plus massifs, comme la présence de la Dharma Initiative qui se fait de plus en plus visible. Les différentes stations Dharma deviennent des décors récurrents et les logos de cette institution envahissent l'écran. De plus, Daniel Faraday prend une importance soudaine au sein du récit et, en un sens, incarne le point de vue science-fictif sur l'intrigue de la série. Tout comme les membres de Dharma, Faraday décrit par la science les phénomènes qu'il rencontre et pense qu'il les maîtrise cognitivement. Enfin, au moment où s'accumulent les signes extérieurs de science-fiction, la saison 4 s'articule autour d'un thème majeur du genre, le voyage dans le temps. Ce thème apparait dès l'épisode 3.8, alors que l'épisode suivant s'appelle «Stranger in a strange land ", titre d'un grand classique de la science-fiction des années 1960, qui pointe d'ailleurs à nouveau vers la question de la spiritualité.

7 Est-on pourtant réellement dans de la science-fiction? En réalité, il faut attendre la fin de la série pour comprendre que la démarche adoptée est bien davantage celle de la fantasy et qu'elle implique un rapport différent aux éléments fictionnels qui divergent par rapport à notre univers. Dès lors, certaines questions deviennent caduques parce que l'enjeu se trouve être ailleurs. Ainsi, personne ne demanderait, à propos d'une œuvre de fantasy, la raison pour laquelle elle met en scène des dragons, car cet élément n'est pas problématique dans cet univers fictionnel. Si Lost relève de la fantasy, alors il n'y a pas lieu de se demander pourquoi l'Homme sans Nom est un monstre de fumée noire ou que certains personnages ont des pouvoirs. En science-fiction, il serait fondamental de se poser cette question parce que l'univers fictionnel serait construit de manière à ce que ces éléments puissent exister et, d'autre part, parce que ces éléments constitueraient l'enjeu de la construction de cet univers. En fantasy, ce qui va compter, ce sont les interprétations symboliques que l'on peut avoir de ces éléments et comment ils s'inscrivent dans un système de représentations, et éventuellement dans un système de valeurs, telles que l'opposition entre le noir de l'Homme sans Nom et le blanc de Jacob.

Or, de manière inhabituelle, ces éléments ont été présentés d'abord comme problématiques alors que souvent, en fantasy, ils sont immédiatement avancés comme non problématiques. Dès lors, ces identités génériques successives relèvent à chaque fois de l'adoption d'un certain point de vue sur un même objet. Le point de vue fantastique est celui de survivants qui vivent dans l'angoisse face à l'inconnu; le point de vue science- 
fictif est porté par ceux qui sont rattachés à la Dharma Initiative ; enfin le point de vue fantasy est celui des Autres et, finalement, des survivants qui ont acquis une connaissance de l'île. Ce qui change n'est pas l'objet mais la perspective. Ainsi, l'Homme sans Nom est qualifié de «monstre » par la plupart des personnages, jusqu'à ce que Danièle Rousseau le qualifie, dans la saison 2, de "système de sécurité ».

9 À travers ces points de vue successifs, le travail sur l'identité générique n'est pas un jeu vide mais résonne avec le propos méta-fictionnel et métaphysique de la série. Il s'agit de modifier le regard en nous montrant comment il est conditionné par un certain nombre d'horizons d'attente et de conceptions a priori. Comme l'écrit Pacôme Thiellement : « C'est le pari de Lost: transformer notre manière d'être en transformant notre manière de regarder; introduire les hommes d'aujourd'hui à une relation consciente au monde de l'âme ${ }^{5}$ ». À partir du moment où les spectateurs adoptent un regard de fantasy, il devient évident que la fantasy a toujours été le genre de la série. Le travail sur l'archétype qui mène à une lecture symbolique est, en effet, à l'œuvre dès le pilote, notamment autour de Locke et du backgammon.

Il n'est, dès lors, pas étonnant de retrouver un grand nombre de récits métaphoriques, presque des paraboles, dans Lost, notamment autour de la représentation des animaux (par exemple, la comparaison entre Charlie et le papillon dans S01E16). Ces éléments relèvent parfois du conte et parfois du mythe - mythe qui devient récit fondateur autour de l'histoire de Jacob et de son frère.

Dans la dernière partie de la série, le voile est levé et, à une géographie imaginaire de l'île qui était d'abord celle de la survie puis celle de la cartographie Dharma ponctuée par les stations, se substitue une géographie fondée sur des lieux d'un passé bien plus ancien, sur des espaces mythiques et sacrés tels que le temple, la statue, le phare et, bien sûr, la caverne de lumière. Trois modalités fondamentales apparaissent alors avec insistance : le mythique, le tragique et l'épique, mettant en valeur ce qui constitue le cœur de cette fiction, l'idée de communauté et de l'être ensemble. Toutes les divergences qui opposent les personnages relèvent de visions différentes posées sur le monde et la vie; si elles ne finissent pas par toutes coïncider, elles parviennent, à terme, à coexister.

Ces différentes modalités invitent à penser de manière figurée la condition humaine dans ce qu'elle a de plus primordiale mais non sous le signe d'un combat manichéen entre le bien et le mal. Il s'agit plutôt de l'affrontement entre deux choix, celui de Jacob et celui de son frère, deux visions de l'ontologie humaine. Il n'y a pas de bien pur puisque Jacob est déjà lui-même corrompu par le crime. Les personnages les plus anciens, les plus puissants, ceux qui semblent appartenir au mythique sont eux-mêmes déterminés par les événements de leur vie, ce qui renvoie la question de l'origine à un passé infini. Cette réinscription dans un passé lointain qui n'est lui-même pas tout à fait le début renvoie précisément à un temps mythologique d'avant l'histoire. Dès lors, le gardien de l'île guérisseur, le conseiller immortel, le medium qui entend les morts ou le jumeau transformé en fumée noire ont tous leur place dans un univers archétypal qui représente, telle une allégorie, l'échiquier de la condition humaine. Pour simplifier, nous passons de la jungle à la station Dharma et de la station Dharma au Temple, haut lieu du symbolique. L'arsenal mystérieux de l'île n'est pas à voir comme un gadget mais comme une entrée vers un espace-temps où tout ce qui fait l'humanité et le monde va être exacerbé.

L'espace de l'île fonctionne dès lors comme microcosme ou image du macrocosme : l'île est une figure de la totalité qui englobe l'ancien, le moderne, le scientifique et le religieux. Le fait que des éléments qui paraissent extérieurs à l'île, comme la trappe dans la saison 2 
ou le village dans la saison 3, soient rétablis dans une continuité avec le reste renforce cette idée de totalité. Alors que nous croyons être sortis de l'île, nous y sommes encore. Cette figure de la totalité fait de l'île une image au sens fort, c'est à dire un lieu légèrement décalé par rapport à notre appréhension quotidienne du monde mais qui, par ce décalage, peut justement éclairer le quotidien.

\section{Changer de perspective}

Revoir la série à la lumière de la fin permet de prendre conscience que ce fonctionnement symbolique de la fiction est présent dès les premiers épisodes. Dans la séquence prégénérique de S01E05, un flashback de l'enfance de Jack est suivi d'une séquence sur l'île où il tente de sauver une personne en train de se noyer, avant de s'apercevoir qu'il s'agit de Boone, qui tentait lui-même de sauver une jeune femme. Cette scène est paradigmatique du fonctionnement de la série puisqu'elle propose une première lecture (une première personne à sauver) pour faire apparaître par la suite un problème plus lointain (la seconde personne en train de se noyer), qui était d'abord caché et que l'on échouera à résoudre. Jack ne parvient pas, en effet, à sauver la jeune femme parce qu'il choisit de ramener d'abord Boone sur le rivage. Comme dans beaucoup de séquences prégénériques de la saison 1, il est frappant de constater que le surnaturel ou les énigmes de l'île sont totalement absents. La séquence parle du personnage qui va être au centre de l'épisode et pose sa problématique en termes de parcours psychologique. Il s'agit ici de l'ambivalence de Jack envers la responsabilité et l'action : il est le héros qui croit qu'il ne peut pas être un héros. Cet aspect de sa personnalité est lié au rapport qu'il entretient avec son père, ce que l'épisode révèle par la suite. Ainsi, dès la saison 1 , c'est bien l'itinéraire initiatique des personnages qui est mis en valeur en pré-générique - et non pas les énigmes à résoudre, sur lesquelles se sont pourtant concentrées les réceptions (et critiques) de la série. L'île est une matérialisation d'un espace symbolique et c'est en arpentant cet espace symbolique (moyen) que les personnages vont pouvoir se découvrir eux-mêmes (but). Plus précisément, l'île est l'espace du symbolique et représente le spirituel. La série montre la tension entre deux voies, celle de la négation du spirituel, représentée par l'Homme sans Nom, et celle de la toute-puissance du spirituel, incarnée par Jacob. Or, l'enjeu de Lost est d'ouvrir une troisième voie, représentée notamment par Jack et Hurley, voie spirituelle médiane et plus humaine, qui met l'amour au centre de la spiritualité.

La série se déploie comme un triptyque : la saison 1 et la saison 6 fonctionnent l'une par rapport à l'autre, la première étant la saison de l'exposition et de la solitude, et la dernière, celle de la résolution et de l'amour. Les saisons 2 à 5 sont les saisons de l'itinéraire initiatique. Cette structure est essentielle parce qu'elle souligne que les véritables enjeux de la série étaient en place dès le commencement, alors que l'attention des spectateurs était encore ailleurs. De manière réflexive et symbolique, dès la première séquence du pilote, Jack se trouve dans la forêt de bambous, juste à côté de la source de lumière de l'île, qu'il ne trouvera qu'à la fin de la série, lorsqu'il sera prêt à la voir. Lors du deuxième visionnage de la série, les spectateurs en savent suffisamment pour prendre les signes extérieurs pour ce qu'ils sont - des éléments de surface, voire des fausses pistes. Fondamentalement, la fin de la série dépeint un monde qui est de l'ordre non pas de l' énigme mais du mystère au sens fort. 
Lost ne fonctionne finalement pas selon le régime de l'indice. Les spectateurs en sont, en fait, prévenus à plusieurs reprises, dans des scènes très didactiques. Ainsi, la séquence pré-générique de S03E07, « Not in Portland », est saturée d'indices qui laissent à penser que l'action se situe sur l'île : la plage, l'aspect du couloir avec la lumière qui fonctionne mal, le vinyle, Ethan, le dialogue où la sœur pourrait sembler parler de Ben, la musique angoissante qu'on attache aux Autres. Mais lors du lever de rideau final, Juliet dévoile littéralement le fait qu'il s'agit de Miami et donc, comme le souligne Aurélie Villers ${ }^{6}$, d'un flashback. Les spectateurs sont tombés dans le piège : ils ont décrypté ce qui leur semblait être des indices mais ont laissé de côté le seul signe auquel ils auraient dû être attentifs : la coiffure de Juliet, qui est celle qu'elle arbore dans les flashbacks. De manière microstructurelle, la série nous montre ici ce qu'il ne faut pas faire pour la comprendre.

Lost joue souvent sur ce procédé de retournement et de transformation didactique du point de vue, en particulier en début de saison, moment clef de la sérialité. Ainsi, dans l'ouverture de la saison 2, tout est fait pour désorienter les spectateurs. En dehors de l'élément récurrent qui ouvre la séquence - l'œil en gros plan - rien ne permet encore aux spectateurs d'identifier le lieu, l'époque ou le personnage. Non seulement l'information est volontairement fragmentaire puisque le personnage est de dos et que l'abondance de gros plans empêchent d'obtenir une vue d'ensemble, mais surtout elle met en avant une série d'indices qui mènent à une fausse piste. La lampe et les vinyles laissent penser que la scène se situe dans les années 1970. À partir du moment où le motif des injections dans le bras crée un effet d'étrangeté, une explosion retentit et la musique change. Ce revirement permet de retrouver l'identité de Lost mais dans un contexte déplacé qui reste encore incompréhensible. Le plan final de la séquence propose un mouvement de caméra étonnant : il s'agit d'une caméra subjective qui se met à la place non pas du corps du personnage de Desmond mais à la place de son regard. Littéralement la caméra suit les ondes lumineuses que son œil capte depuis la lunette jusqu'aux miroirs réfléchissants et jusqu'à ce qu'il voit. Ce mouvement de caméra peut se lire comme une matérialisation de la question du regard, du point de vue et de la perspective. Dans le retournement final, les spectateurs prennent conscience non seulement de la continuité mais de la simultanéité entre cette scène d'ouverture et la scène finale de la saison $1:$ les deux se sont passées au même moment, mais selon deux points de vue différents, celui du groupe et celui de Desmond. Un début de saison consiste précisément à re-commencer, c'est à dire à s'inscrire dans une continuité mais aussi à se renouveler. C'est ce que fait Lost en se réinventant de manière inattendue. La série n'évolue pas de manière linéaire mais en ajoutant une strate plus profonde à ce qu'elle avait déjà présenté. Par conséquent, ce n'est pas un hasard si cette ouverture de saison se déroule sous terre, puisqu'il s'agit de creuser plus profondément le réel.

Ce procédé utilise les possibilités de la séquence pré-générique en tant que séquence décontextualisée, puisque rien ne la précède en début d'épisode et $a$ fortiori en début de saison. Le travail des spectateurs est donc toujours de se resituer par rapport à ce qu'ils voient. Il s'agit d'adopter dès lors une posture herméneutique ; il faut tenter d'interpréter les signes fournis, alors que ne sont jamais donnés les indices qui permettraient de comprendre réellement de quoi il s'agit avant le coup de théâtre final. En effet, il faut que les spectateurs soient passés par un chemin pour pouvoir accéder à un autre niveau de compréhension. De la même manière, l'ouverture de la saison 6 refonde entièrement ce que l'on croyait savoir dans un mouvement de caméra qui offre un plan spectaculaire et un changement de perspective similaires à ceux des ouvertures des saisons 2 et 3 . Cette 
fois, la caméra plonge au lieu de s'élever, découvrant l'île engloutie au fond de l'océan. Il s'agit là d'un ultime retournement, un retournement du retournement : ce qu'il nous faut faire n'est donc peut-être pas d'élever le regard comme dans S02E01 et S03E01 dans un mouvement surplombant qui offrirait une vue en partie obstruée (car nous regarderions toujours d'en bas quelque chose qui nous échappe), mais il nous faut plutôt regarder vers le bas, c'est à dire creuser ce que nous avons déjà sous les yeux.

Par conséquent, le chemin de la série est une expérience initiatique pour les personnages comme pour les spectateurs. Chaque personnage se fait herméneute à un moment ou à un autre et fonde sa vision du monde sur une interprétation des signes. Or, tous finissent par se tromper. Lorsque nous découvrons Daniel Faraday, nous avons l'impression que le personnage maîtrise son sujet et nous adoptons donc naturellement son point de vue. «Je sais ce qu'il se passe » («I know what's happening »), dit Daniel dans S05E01. Il montre alors son carnet et indique que celui-ci contient tout ce qu'il a appris par l'étude de la physique sur le temps mais aussi tout ce qu'il a appris sur la Dharma Initiative. Pourtant son regard est réducteur, comme celui de la Dharma Initiative d'ailleurs. C'est la raison pour laquelle il se trouve finalement dépassé par les événements : il va devoir dire à Charlotte enfant ce qu'il ne voulait pas lui avouer et même être tué par sa mère. Son action est donc un échec complet, parce que ces événements se jouent sur un plan qui n'est pas le sien cognitivement. Son point de vue ne permet pas d'appréhender la totalité des phénomènes. Ce n'est pas parce que son action est parfois efficace qu'elle est conduite à partir de prémisses vraies. C'est exactement ce qui se passe pour les spectateurs qui se plaçaient du côté de l'indice. À la fin de la série, ce mode d'appréhension de la fiction s'avère impossible. Il ne fonctionne pas dans ce cadre. La seule solution est de changer de perspective, c'est à dire accepter de ne pas être dans un récit de science-fiction. L'enjeu de la série est donc de se libérer des conditionnements. Dès lors que l'on accepte de changer de regard, il est possible d'expérimenter réellement la série. Cela n'implique pas de nier tout ce qui a précédé. Au contraire, le chemin est aussi important que le but. C'est parce que l'on a expérimenté ces multiples changements de points de vue, cette fiction kaléidoscopique, que l'on peut finalement remettre en question les évidences et nos propres conditionnements.

20 Par conséquent, les notions d'écho et de résonance sont constantes dans Lost : maintes fois, un personnage prononce une phrase que son interlocuteur n'est pas encore prêt à entendre et qu'il ne comprendra que bien plus tard. Ainsi, à la fin de la cinquième saison, Rose et Bernard résument à eux seuls, et en quelques phrases très simples, l'enjeu de la série. À ce stade, les spectateurs sont naturellement portés vers le point de vue de Sawyer et de Kate, qui ne sont pas (encore) capables d'appréhender la portée du discours tenu par Rose et Bernard. Or, cette scène anticipe, de très loin, la toute fin de la série. Voici ce que dit Bernard dans S05E16: "Eh bien, on meurt. Tout ce que nous voulons, c'est être ensemble. C'est tout ce qui compte finalement » («So, we die. All we care is being together. It's all that matters in the end »). Ce que le couple a déjà saisi, les autres survivants ne le comprendront que bien plus tard.

\section{Un ensemble show}

De fait, les personnages se construiront un monde de l'après, un espace hors temps, pour se retrouver après la mort. Ce qui compte in fine, c'est d'être ensemble. C'est pourquoi la série est fondamentalement un ensemble show, non pas dans la juxtaposition des 
itinéraires mais dans la construction du groupe, dans les liens qui se tissent au fur et à mesure des saisons. Non seulement les flashbacks des premières saisons révèlent l'infinité de liens qui s'étaient tissés entre les personnages avant leur rencontre, comme les fils de la tapisserie de Jacob, mais, une fois sur l'île, amitiés et amour sont au cœur des intrigues. Tel un soap opera (ou une romance shakespearienne comme le montrent Sarah Hatchuel et Randy Laist), Lost offre des rebondissements constants, la découverte de filiations cachées, des triangles amoureux, des drames à n'en plus finir. Finalement il faudrait interroger l'articulation qui s'opère dans la série entre la fantasy et le mélodrame. Dans le cadre de la fantasy, l'aspect paroxystique et primordial des intrigues mélodramatiques vont prendre leur ampleur et leur universalité. C'est cette dimension qui constitue finalement le point d'aboutissement ultime de la série. Dès le début, il est clair que les relations entre les personnages vont porter l'intrigue, tout autant, si ce n'est plus, que la construction de la mythologie (car la mythologie est fondée sur l'idée même de l'amour).

Dès le début, la série multiplie les scènes dramatiques, notamment en fin d'épisodes : des scènes muettes sur une chanson ou un morceau de Michael Giacchino, des scènes de retrouvailles sur la plage ou des moments d'échanges entre la vie et la mort comme la séquence paradigmatique du décès de Boone en parallèle avec la naissance d'Aaron. Comme l'écrit Sarah Hatchuel :

Parce que l'un de ses thèmes phares est la rédemption à travers une communauté formée par des souffrances et des joies communes, le deuil, la solidarité, l'amitié et l'amour, Lost présente une réflexion sur ce que signifie « vivre ensemble», sur les liens entre les individus et sur la construction de l'identité et de l'altérité?.

L'itinéraire des personnages et des spectateurs consiste à lâcher prise («let go »), c'est à dire à accepter que l'on ne puisse pas tout maîtriser pour pouvoir expérimenter le monde et la fiction. Le fonctionnement des flashbacks reflète cette idée. Un des principes essentiels du montage est la présomption de pertinence: le cerveau crée des liens, notamment des liens de causalité, entre les plans que nous voyons. C'est ainsi que nous avons appris à lire une image et à construire du sens. Ainsi, le flashback est perçu naturellement comme expliquant ce qui se passe dans la ligne narrative principale. Or l'un des grands enjeux de la série est de couper le lien causal, c'est à dire non pas de nier le déterminisme, mais de se libérer de son poids. On retrouve ici une nouvelle structure en trois temps: d'abord, l'époque des flashbacks, c'est à dire du sur-déterminisme du passé, qui vient hanter le présent comme un poids, puis le temps des flashforwards : par un renversement de perspective, c'est la ligne narrative principale qui devient le passé hantant les personnages dans l'avenir (ainsi, Jack veut revenir sur l'île). Enfin, un troisième temps se dessine avec la saison 6 : soudain, les séquences n'ont plus de lien causal avec la ligne narrative principale, à tel point qu'on a pensé à des flash-sideways, à une réalité parallèle qui aurait été justement déconnectée de la ligne principale. En réalité, il s'agit de flash-ahead, d'un hors temps destiné à ce que les personnages se retrouvent après la mort. Il s'agit bien de la saison du «let go ». Tout ce qui s'est passé conduit à ce moment, non plus comme un poids de la nécessité subie.

Les flash-ahead constituent un monde construit par les personnages, un monde à leur image. Il s'agit donc d'une question de perspective. Ce n'est pas que le tragique n'est plus là (Locke est paralysé, Charlie est drogué, le père de Jack est mort). Ce n'est pas que la souffrance est absente, au contraire. Il s'agit simplement de la percevoir autrement, de 
l'accepter et de la vivre ensemble. La formule de Christian Shepard à la fin de la série, est "se souvenir et lâcher prise» («to remember and let go»). Et pour cela, il faut expérimenter un contact réel avec autrui, le partage d'une émotion. Tel est le sens des anamnèses qui rythment la fin de la série. Au moment où les personnages sont prêts, l'anamnèse représente l'instant où ils se réapproprient leur vie, où ils réinvestissent leur parcours. Il s'agit de faire retour mais dans l'apaisement. Ce qui est mis en avant ici est moins la connaissance que la re-connaissance : ce moment où les deux personnages se reconnaissent, c'est à dire retrouvent leur propre identité par contact avec l'autre, dans une illustration littérale de l'expression « to remember and let go ».

Ce que montre Lost, c'est que même s'il est impossible de connaître tous les faits, il est, en revanche, toujours possible d'aimer. Finalement c'est cet amour qui donne accès à une forme de vérité supérieure, que cet amour prenne la forme du couple, de l'amitié, ou de l'amour universel représenté par Hurley, qui aime et aide tout le monde. Ce n'est donc pas un hasard si ce dernier finit gardien de l'île. L'enjeu essentiel et existentiel de Lost n'est autre que cette reconnaissance. La scène où Ilana dit à Ben « Je t'accepte » («I'll have you ", S06Е07) définit cet enjeu: elle l'accepte officiellement dans le groupe. La construction paradoxale de Lost, qui est à la fois si cérébrale et complexe et en même temps si centrée sur l'émotion, se résout ainsi. Toute la structure mène à comprendre ce que peut être la vie d'un être humain. Il nous fallait céder au mystère, entrer dans l'allégorie, entrer dans la fiction. Il nous fallait lever un à un les voiles, découvrir les différentes facettes de l'image pour en comprendre la complexité et la simplicité. Il fallait transformer notre regard pour que nous puissions à la fois revenir sur la série et sur notre vie et les voir autrement. Toute la complexité du travail générique se résout dans la simplicité d'une forme primordiale qui a à voir avec le mythique, le merveilleux, l'empathie et le vivre ensemble. Le travail que fait Lost est un travail typiquement sériel parce qu'il repose non pas sur l'entrée dans la fiction mais sur la récurrence, la variation et surtout le retour. Il ne s'agit pas de commencer, mais de s'offrir la chance de recommencer, au moins symboliquement, de saisir l'opportunité, quel que soit le point dont on part, d'un nouveau regard.

\section{BIBLIOGRAPHIE}

CORNILLON Claire, Par-delà l'infini, La spiritualité dans la science-fiction française, anglaise et américaine, Thèse de doctorat en Littérature comparée, sous la direction de Jean Bessière, Université Paris 3 - Sorbonne nouvelle, 2012, https://tel.archives-ouvertes.fr/tel-00869974

HATCHUEL Sarah, Lost : Fiction vitale, Paris, Presses universitaires de France, 2013.

MELLIER Denis, L'Ecriture de l'excès, Fiction fantastique et poétique de la terreur, Paris, Champion, 1999.

THIELLEMENT Pacôme, Les Mêmes yeux que Lost, Paris, Léo Scheer, 2011.

TODOROV Tzvetan, Introduction à la littérature fantastique, Paris, Points, 2015 [1970]. 
VILLERS Aurélie, "Récits analeptiques et distanciation cognitive temporelle dans la série Lost », communication présentée au colloque de Cerisy « Comment rêver la science-fiction à présent ? ", 2009.

\section{NOTES}

1. Cet article reprend et poursuit la réflexion entamée dans un article journalistique que j'avais rédigé en 2009 pour le site d'actualité culturelle Lintermede.com : http://www.lintermede.com/ series-lost-jj-abrams-damon-lindelof-jeffrey-lieber.php

2. Voir Claire Cornillon, Par-delà l'infini, La spiritualité dans la science-fiction française, anglaise, et américaine, thèse de doctorat en littérature comparée, sous la direction de Jean Bessière, Université Paris 3 - Sorbonne nouvelle, 2012, https://tel.archives-ouvertes.fr/tel-00869974

3. Voir Tzvetan Todorov, Introduction à la littérature fantastique, Paris, Points, 2015 [1970].

4. Sur le fantastique de la sidération, voir Denis Mellier, L'Ecriture de l'excès, Fiction fantastique et poétique de la terreur, Paris, Champion, 1999.

5. Pacôme Thiellement, Les Mêmes yeux que Lost, Paris, Léo Scheer, 2011, p. 53.

6. Aurélie Villers, "Récits analeptiques et distanciation cognitive temporelle dans la série Lost ", communication présentée au colloque de Cerisy « Comment rêver la science-fiction à présent ? ", 2009.

7. Sarah Hatchuel, Lost : Fiction vitale, Paris, Presses Universitaires de France, 2013, p. 95.

\section{RÉSUMÉS}

Lost semble, à première vue, présenter un glissement générique du fantastique à la fantasy, en passant par la science-fiction mais révèle finalement un fonctionnement didactique qui invalide une série d'hypothèses cognitives et de modes de lecture de la série. In fine, c'est la reconnaissance plus que la connaissance qui semble au cœur de la série : plutôt qu'une énigme, la série est un mystère qu'il faut accepter et qui confronte à l'expérience d'une communauté d'amour et d'acceptation.

Lost seems at first to present an evolution from the fantastic to fantasy, through science fiction, but eventually reveals a didactic pattern that invalidates a series of cognitive hypotheses and viewpoints on the series. In fine, acceptance is more important in Lost than knowledge: rather than an enigma, the series is a mystery that we have to accept and an experience of a community of love and acceptance.

\section{INDEX}

Mots-clés : Lost, narration, genre, science-fiction, fantastique, code

Keywords : science fiction, fantastic, fantasy, codes 


\section{AUTEUR}

\section{CLAIRE CORNILLON}

Claire Cornillon est docteur en littérature comparée. Elle est également titulaire d'un master en études cinématographiques. Elle est actuellement PRAG à l'Université Paul Valéry-Montpellier 3. Ses recherches les plus récentes portent sur la narration et la fiction dans les séries télévisées américaines, en particulier de network. Elle co-dirige la collection Sérial aux Presses Universitaires François Rabelais.

Claire Cornillon has a PhD in comparative literature and a master in film studies. She teaches at the Université Paul Valéry-Montpellier 3. Her latest research aims at studying fiction and narration in American TV series, especially network series. She is general co-editor of the «Sérial » collection published at the Presses Universitaires François Rabelais. 